\title{
Do All Patients with Newly Diagnosed Prostate Cancer Need Staging Radionuclide Bone Scan? A Retrospective Study
}

\author{
Mohammed A. Al-Ghazo, Ibrahim F. Ghalayini, Rami S. al-Azab, Ibrahim Bani-Hani, Alaa \\ Barham, Yazan Haddad
}

Faculty of Medicine (MAA, IFG, RSA, IBH), King Abdullah University Hospital, Jordan University of Science and Technology, Irbid, Jordan and Princess Haya Biotechnology Center (YH), Jordan University of Science and Technology, Jordan

\begin{abstract}
Purpose: Define a group of patients with newly diagnosed prostate cancer, whose risk of bone metastasis is low enough to omit a bone scan staging study.

Materials and Methods: From 2003 to 2009, the medical records of patients who were newly diagnosed with prostate cancer were retrospectively reviewed. The data collected included: age, digital rectal examination, serum prostate specific antigen (PSA), Gleason score, clinical T stage, and bone isotope scan. Patients were divided into two groups according to the results of bone isotope scan; positive group and negative group. A univariate and multivariate binary logistic regression was used to analyze the results.

Results: Of the 106 patients, 98 had a complete data collection and were entered into the study. The median age of the patients was 70.5 years and patients with a positive bone scan was 74 years, significantly higher than for patients with negative scans (69 years) $(\mathrm{p}=0.02)$. Bone metastasis was detected in 39 cases $(39.7 \%)$. In all patients with clinical T1-2 stage, a Gleason score of $<8$ and PSA $\leq 20 \mathrm{ng} / \mathrm{mL}$, the bone isotope scans were negative. In univariate analysis, PSA ( $>$ $20 \mathrm{ng} / \mathrm{mL}$ ) and Gleason score (>7) were independently predictive of positive bone scan, while clinical stage was not. Conclusion: Staging bone scans can be omitted in patients with a PSA level of $\leq 20 \mathrm{ng} / \mathrm{mL}$, and Gleason score $<8$. Our results suggest that by considering the Gleason score and PSA, a larger proportion of patients with prostate cancer could avoid a staging bone scan.
\end{abstract}

Key words: prostate-specific antigen; prostatic neoplasms; Gleason score; bone scan

Int Braz J Urol. 2010; 36: 685-92

\section{INTRODUCTION}

Prostate cancer is one of the most commonly diagnosed malignant diseases in Western countries but it is relatively rare in Asia (1). Prostate specific antigen (PSA) is a valuable tool for detecting prostate cancer (PC), but it is not perfect. The test lacks both the sensitivity and specificity to accurately detect the presence of PC. PSA is a prostate-specific marker, not a PC marker (2). Since the dosing of serum PSA is accompanied by failure, variants of PSA measurements have been described in order to increase its accuracy: free/total PSA ratio, age-specific PSA, PSA density and PSA velocity (3). It was concluded that the combination of the PSA density, free PSA percentage and age are factors which contribute to high accuracy for PC detection (4). Despite the development of tools that help early diagnosis of organ-confined prostate 
cancer, up to $22 \%$ of newly diagnosed patients has been reported to have advanced or metastatic disease (5). The accurate staging of newly diagnosed patients helps to assess prognosis and ensure appropriate treatment. The staging process for patients diagnosed with prostate cancer includes a digital rectal examination (DRE), PSA, as well as pelvic computed tomography (CT) scan, pelvic lymphadenectomy and radionuclide bone scan (6). The detection of bone metastases using isotope bone scanning was first described using strontium in the early 1960s and it has long been the standard reference investigation for detecting bone metastases in prostate cancer (7). Technetium-99m labeled diphosphonates is very sensitive but its specificity is reduced in older men (7). Bone is the second most common site for metastatic deposits from prostate cancer and the skeleton is involved in $80-85 \%$ of patients who die of prostate cancer (8). Patients with prostate cancer are not at the same risk of developing bone metastasis. This may be translated into a high number of patients where staging bone scan studies can be avoided with a significant reduction of costs for the health care system. The American Urological Association (AUA) (9) and the European Association of Urology (EAU) (10) guidelines for prostate cancer suggest that bone scan may not be indicated in asymptomatic patients if serum PSA level is less than $20 \mathrm{ng} / \mathrm{mL}$ in the presence of well differentiated tumor, while the Japanese Urological Association guidelines for prostate cancer in 2006 indicates that a bone scan can be omitted for patients with PSA level of $10 \mathrm{ng} / \mathrm{mL}$ or less who have well differentiated prostate cancer (11). Although the issue remains controversial and the AUA and EUA guidelines for prostate cancer are still externally not completely validated, in this study we retrospectively tried to evaluate the relationship between clinical T stage, serum PSA level, Gleason score and bone metastasis, in an attempt to define a group of patients whose risk of a positive radioisotope bone scan is low enough to be omitted.

\section{MATERIALS AND METHODS}

From 2003 to 2009, the medical records of patients who were newly diagnosed with prostate cancer at King Abdullah University Hospital were retrospectively reviewed. The data collected included the following information: age, DRE, serum PSA, transrectal ultrasound of the prostate, prostatic biopsy Gleason score, clinical T stage, bone pain, and bone radioisotope scan. Data regarding prostate biopsy core involvement (number from each side and percentage of each core) were not analyzed because pathological reports of many patients had incomplete information. Of this cohort, patients with prior treatment, including hormonal therapy, radical prostatectomy, or radiation therapy were excluded. Patients were included only if they had their PSA estimated within 4 weeks of the bone scan. Patients were divided into two groups according to the results of bone isotope scan; positive group and negative group. Serum PSA levels were usually obtained using the Hybritech assay (upper limit of $4 \mathrm{ng} / \mathrm{mL}$ ). T staging was based on the results of DRE because information regarding transrectal prostate ultrasound, CT scan, magnetic resonance imaging or biopsies was not used because it was not available in all patients' medical records. The results of the Tc- $99^{\mathrm{m}}$ methylenediphosphonate radionuclide bone scan were obtained from the radiology reports (by a full-time radiologist in nuclear medicine) from the database of the hospital. For patients with equivocal bone scans a CT scan was performed to confirm the isotope scan findings.

In order to establish criteria for avoiding bone scans in prostate cancer; a univariate and multivariate binary logistic regression approach was used to measure odds ratios for age, PSA levels, Gleason score, and $\mathrm{T}$ staging. Multivariate results were based on direct entry of chosen variables because the stepwise selection model only accepted the PSA levels as a significant model. The differences in significance of subgroups in the studied variables were used as cutoff criteria for avoiding bone scans in prostate cancer. Categorical variables were compared according to bone scans using Pearson's chi square test. A p-value $<0.05$ was considered significant. All tests were performed on SPSS 16.0 software.

\section{RESULTS}

Of the overall 106 patients with newly diagnosed prostate cancer, 98 had a complete retrospective 
Table 1 -Demographic and clinical data in both groups of patients with prostate cancer.

\begin{tabular}{lccc}
\hline Patients Characteristics & Bone Metastasis & p Value \\
& Present & Absent & \\
\hline Age (years) & 4 & 16 & 0.04 \\
$\quad<65$ & 16 & 27 & \\
$65-75$ & 19 & 16 & \\
$>75$ & & & \\
PSA & 5 & 27 & \\
$\quad<10(\mathrm{ng} / \mathrm{mL})$ & 2 & 18 & \\
$10.1-20(\mathrm{ng} / \mathrm{mL})$ & 8 & 7 & \\
$20.1-40(\mathrm{ng} / \mathrm{mL})$ & 12 & 4 & \\
$40.1-99(\mathrm{ng} / \mathrm{mL})$ & 12 & 3 & \\
$\geq 100(\mathrm{ng} / \mathrm{mL})$ & & & \\
Gleason score & 7 & 26 & \\
$2-6$ & 7 & 12 & 0.1 \\
7 & 25 & 21 & \\
$8-10$ & & & \\
Clinical stage & 18 & 40 & \\
T 1-2 & 21 & 19 & \\
T3-4 & & & \\
\hline
\end{tabular}

PSA = prostate specific antigen .

data collection and were entered into this study. The median age of the patients was 70.5 years and patients with a positive bone isotope scan was 74 years, significantly higher than the median age for patients with negative scans (69 years) $(\mathrm{p}=0.02)$. Demographic and clinical data for both groups of patients (patients with positive scans and patients with negative scans) are shown in Table-1.

Gleason score of 8-10 was found in 45 out of 98 patients $(45.9 \%)$, and clinical T1-2 stage was found in 61 out of 98 cases $(62.2 \%)$.

Bone metastasis was detected in 39 cases (39.7\%). In all patients with clinical T1-2 stage, Gleason score of $<8$ and PSA $\leq 20 \mathrm{ng} / \mathrm{mL}$, bone isotope scans were negative, while four out of 12 patients $(33.3 \%)$ with $\mathrm{T} 1-2$, PSA $<10 \mathrm{ng} / \mathrm{mL}$ and Gleason score $8-10$ had positive bone metastasis as seen in Table-2.

Table-3 demonstrates the univariate and multivariate analyses to assess the ability of age, PSA,
Gleason score and clinical $\mathrm{T}$ stage to predict a positive bone isotope scan.

In univariate analysis, PSA (values of PSA greater than $20 \mathrm{ng} / \mathrm{mL}$ ) and Gleason score ( $>7$ ) were independently predictive of positive bone scan, while clinical stage was not. Age was only significant for those people who were older than 75 years.

In multivariate analysis, PSA was significant regardless of the other adjusted factors. The strongest predictor of a positive scan was a serum PSA of $>100 \mathrm{ng} / \mathrm{mL}$ (Table-3). Interestingly, serum PSA levels of $\leq 20 \mathrm{ng} / \mathrm{mL}$ did not independently predict a positive scan on multivariate analysis. Age lost the significance value in the multivariate analysis because of the effect of other factors (PSA, Gleason score and clinical T stage). In general the multivariate analysis showed a decrease in the significance levels and in the odds ratios (OR) with the exception of the PSA range of 20.1-40, which had an increase in OR. 
Prostate Cancer and Radionuclide Bone Scan

Table 2 - Number of positive bone isotope scan in relation to PSA, Gleason score and T stage prostate cancer.

\begin{tabular}{lcccccc}
\hline PSA $(\mathrm{ng} / \mathrm{mL})$ & \multicolumn{3}{c}{$\begin{array}{c}\text { Clinical Stage T1-T2 } \\
\text { Gleason score }\end{array}$} & \multicolumn{3}{c}{$\begin{array}{c}\text { Clinical Stage T3-T4 } \\
\text { Gleason score }\end{array}$} \\
\hline & $2-6$ & 7 & $8-10$ & $2-6$ & 7 & $8-10$ \\
$<10$ & $0 / 12$ & $0 / 5$ & $2 / 6$ & $0 / 4$ & $0 / 1$ & $3 / 4$ \\
$10.1-20$ & $0 / 5$ & $0 / 4$ & $2 / 6$ & $0 / 2$ & $0 / 1$ & $0 / 2$ \\
$20.1-40$ & $2 / 2$ & $0 / 0$ & $1 / 4$ & $2 / 4$ & $0 / 0$ & $3 / 5$ \\
$40.1-99$ & $1 / 1$ & $2 / 3$ & $4 / 5$ & $0 / 1$ & $1 / 1$ & $4 / 5$ \\
$\geq 100$ & $0 / 0$ & $3 / 3$ & $3 / 5$ & $1 / 2$ & $2 / 2$ & $3 / 3$ \\
\hline
\end{tabular}

PSA = prostate specific antigen.

Table 3 - Analysis of independent factors (age, PSA, Gleason score, clinical T stage) predictive of positive bone metastasis on bone isotope scan.

\begin{tabular}{|c|c|c|c|c|}
\hline \multirow[t]{2}{*}{ Factors } & \multicolumn{2}{|c|}{ Univariate Analysis } & \multicolumn{2}{|c|}{ Multivariate Analysis } \\
\hline & OR $(95 \% \mathrm{CI})$ & p Value & OR $(95 \%$ CI $)$ & p Value \\
\hline \multicolumn{5}{|l|}{ Age (year) } \\
\hline$<65$ & 1 & & 1 & \\
\hline $65-75$ & $2.4(0.7-8.3)$ & 0.2 & $2.1(0.4-10.7)$ & 0.34 \\
\hline$>75$ & $4.8(1.3-17.1)$ & 0.02 & $4.4(0.8-24.2)$ & 0.07 \\
\hline \multicolumn{5}{|c|}{ PSA (ng/mL) } \\
\hline$<10$ & 1 & & 1 & \\
\hline $10.1-20$ & $0.6(0.1-3.4)$ & 0.6 & $0.7(0.1-4.1)$ & 0.6 \\
\hline $20.1-40$ & $0.6(0.1-3.4)$ & 0.01 & $5.5(1.2-26.0)$ & 0.03 \\
\hline $40.1-99$ & $16.2(3.7-71.2)$ & $<0.001$ & $12.5(2.6-60.0)$ & 0.002 \\
\hline$\geq 100$ & $21.6(4.4-100.1)$ & $<0.001$ & $16.0(3.1-83.0)$ & 0.001 \\
\hline \multicolumn{5}{|c|}{ Gleason score } \\
\hline $2-6$ & 1 & & 1 & \\
\hline 7 & $2.2(0.6-7.6)$ & 0.2 & $1.8(0.3-10.1)$ & 0.5 \\
\hline 8-10 & $4.4(1.6-12.2)$ & 0.004 & $3.9(1.1-14.2)$ & 0.04 \\
\hline \multicolumn{5}{|l|}{ T stage } \\
\hline $\mathrm{T} 1-\mathrm{T} 2$ & 1 & & 1 & \\
\hline $\mathrm{T} 3-\mathrm{T} 4$ & $2.0(0.9-4.6)$ & 0.1 & $1.1(0.4-3.4)$ & 0.6 \\
\hline
\end{tabular}

$C I=$ confidence interval; $P S A=$ prostate specific antigen; $O R=$ odds ratio.

\section{COMMENTS}

Initial staging of prostate cancer is necessary for choosing the type of treatment. Bone scanning has been routinely used to detect bone metastases for patients with newly diagnosed prostate cancer (6). However, the detection rate of bone metastases in newly diagnosed prostate cancer is influenced by three major prognostic factors; PSA, stage and grade (12). Although the relationship between PSA and bone scan findings has been investigated, controversy exists as reports remain conflicting (12). Recent guidelines published by the National Institute for Clinical Excellence (NICE) (13) stated that bone scans are not required for staging purposes in prostate cancer with a PSA level of $<10 \mathrm{ng} / \mathrm{mL}$ where the Gleason score is 
$<8$ and no bone pain is present, as there is a very low likelihood of detecting metastatic disease. This recommendation is based on previous studies which reported a high negative predictive value when bone scans were not used in patients with a PSA level of $<10 \mathrm{ng} / \mathrm{mL}$. It was reported that bone scan can be omitted if PSA $<20 \mathrm{ng} / \mathrm{mL}$ (14), other series recommended omitting bone scan for PSA less than $10 \mathrm{ng} / \mathrm{mL}$ (15), whereas Lin et al. (8) concluded that bone scan is indicated in all patients. In this series, baseline bone scan could be omitted in asymptomatic patients with PSA $\leq 20$ $\mathrm{ng} / \mathrm{mL}$ and Gleason score $<8$, which is in agreement with the American Urological Association (9) and European Association of Urology (10) guidelines. In the present study, the bone scan was positive in 7 out of 52 patients (13.4\%). By applying these criteria, this rate of bone metastases at this level of PSA is higher than that previously reported (16), because there were a high proportion of patients with a high Gleason score $\geq 8$. In our series, the rate of bone metastases in patients with PSA $10 \mathrm{ng} / \mathrm{mL}$ or less was $15.5 \%$ (5 out of 32 patients), which is in agreement with reported series (14), while bone metastases was $10 \%$ (2 out of 22 patients) in patients with PSA level 10.1 -20 $\mathrm{ng} / \mathrm{mL}$. This rate was less than the results in reported series (17). In addition, all the seven patients in our study had a Gleason score of 8 or higher. The current results revealed that bone metastases is common in Jordanian patients with newly diagnosed prostate cancer with an overall positive rate of $39.7 \%$ which is higher than that in USA (8.9\%) (8) and Japan $(22.2 \%)(18)$, which is probably due to lack of a screening program in Jordan for prostate cancer and low awareness of patients, as well as the physicians. Several studies attempted to use the Gleason score to further refine the criteria for staging bone scan. Lee et al. (19) evaluated the Gleason score, PSA level and clinical stage by univariate and multivariate analysis for their ability to predict a positive bone scan in 631 consecutive patients with prostate cancer. Multivariate analysis showed that Gleason score, PSA and clinical stage were significant independent predictors for positive scan and the authors suggested that eliminating staging bone scans would be possible in low risk groups (Gleason score 2-7, PSA $\leq 50 \mathrm{ng} / \mathrm{mL}$ and clinical stage $\leq \mathrm{T} 2 \mathrm{~b}$ ). We agree with these authors but based on a lower PSA level $(\leq 20$ $\mathrm{ng} / \mathrm{mL}$ ). In our series, no patient with PSA level $\leq 20$ $\mathrm{ng} / \mathrm{mL}$ and Gleason score less than 8 had a positive bone scan in contrast to those with Gleason score $\geq 8$ and PSA $\leq 20 \mathrm{ng} / \mathrm{mL}$, as $7.1 \%$ had positive scans. This confirms the most recently published series aimed at predicting the presence of bone metastases at diagnosis which indicate that not only PSA but also other clinical variables such as clinical stage and Gleason score should be considered (18). Gleave et al. (20) mentioned that the negative predictive value of clinical $\mathrm{T}$ stage was insignificant to identify the patients with a low risk for bone metastases, which was in agreement with the results in our group of patients. In this series, it could be as a result of the fact that the DRE was done by more than one urologist and the number of patients was small because this study was carried out in a single hospital and the incidence of prostate cancer in Jordan as an Asian countries is less than in Western countries. Compared with the USA, the incidence and age-adjusted mortality rates for prostate cancer in Asian countries can be up to 10-fold lower (21). Probably, the low incidence of prostatic cancer in Asia may be due to genetic, dietary or environmental factors and their lifestyles (22). We believe combination of different factors in patients with newly diagnosed prostate cancer, especially PSA and Gleason score in association with skeletal symptoms are important in predicting bone metastases. Chybowski et al. (23) investigated the ability of local clinical stage, tumor grade (based on Mayo Clinic histological grading system) and serum acid phosphatase to predict radionuclide bone scan findings. Although all these clinical parameters directly predicted the incidence of a positive bone scan on multivariate analysis, PSA was the best overall predictor of bone scan findings which is similar to our findings. In this series, the median age of patients with positive bone scan was higher than those with negative scans which is consistent with other reported series (7). This may reflect at least partly the lead-time for the progression of prostate cancer to involve the skeleton, but it may also reflect differences in patterns of referral and diagnosis.

Some limitations of the present study deserve mention: this study was retrospective, as in all the other series on this subject. We could find no prospective, randomized studies; therefore, urologists must 
judge for themselves as to how much weight should be given to retrospective studies. Furthermore, we cannot exclude the fact, even more accurate prediction of bone metastasis might have been reached if other variables such as the number of prostate biopsy cores involved by tumor from each lobe of prostate and the percentage of each core were analyzed. Additional studies are required to clarify the importance of these variables in relation to skeletal metastases.

\section{CONCLUSION}

Despite some limitations in the present study (this study is a retrospective and there is a lack of data regarding core involvement), we can conclude that routine bone scan for initial staging of prostate cancer is no longer required. A baseline bone scan can be omitted in Jordanian patients with newly diagnosed prostate carcinoma with serum PSA level $20 \mathrm{ng} / \mathrm{mL}$ or less, Gleason score less than 8 and without skeletal symptoms. These criteria, if implemented, would result in fewer bone scans in patients with prostate cancer than recommended by the National Institute for Clinical Excellence - NICE (13) with considerable cost saving. Another benefit of reducing the numbers of bone scans is that fewer patients will have the stress of waiting for their scan results. It is possible that by using these guidelines, very few patients with bone metastases might go undetected against the emotional and financial gains and reducing waiting time for radioisotope scan investigation. Clinical judgment should be used where patients are on the margins of the guidelines.

\section{CONFLICT OF INTEREST}

None declared.

\section{REFERENCES}

1. Chen CH, Tzai TS, Huang SP, Wu HC, Tai HC, Chang $\mathrm{YH}$, et al.: Clinical outcome of Taiwanese men with metastatic prostate cancer compared with other ethnic groups. Urology. 2008; 72: 1287-92.
2. Nogueira L, Corradi R, Eastham JA: Prostatic specific antigen for prostate cancer detection. Int Braz J Urol. 2009; 35: 521-9; discussion 530-2.

3. Martinez CA, Dall'Oglio M, Nesrallah L, Leite KM, Ortiz V, Srougi M: Predictive value of PSA velocity over early clinical and pathological parameters in patients with localized prostate cancer who undergo radical retropubic prostatectomy. Int Braz J Urol. 2004; 30: 12-7.

4. Gregorio EP, Grando JP, Saqueti EE, Almeida SH, Moreira HA, Rodrigues MA: Comparison between PSA density, free PSA percentage and PSA density in the transition zone in the detection of prostate cancer in patients with serum PSA between 4 and $10 \mathrm{ng} / \mathrm{mL}$. Int Braz J Urol. 2007; 33: 151-60.

5. Thurairaja R, McFarlane J, Traill Z, Persad R: State-ofthe-art approaches to detecting early bone metastasis in prostate cancer. BJU Int. 2004; 94: 268-71.

6. Meng E, Sun GH, Wu ST, Chuang FP, Lee SS, Yu DS, et al.: Value of prostate-specific antigen in the staging of Taiwanese patients with newly diagnosed prostate cancer. Arch Androl. 2003; 49: 471-4.

7. O'Sullivan JM, Norman AR, Cook GJ, Fisher C, Dearnaley DP: Broadening the criteria for avoiding staging bone scans in prostate cancer: a retrospective study of patients at the Royal Marsden Hospital. BJU Int. 2003; 92: 685-9.

8. Lin K, Szabo Z, Chin BB, Civelek AC: The value of a baseline bone scan in patients with newly diagnosed prostate cancer. Clin Nucl Med. 1999; 24: 579-82.

9. Thompson I, Thrasher JB, Aus G, Burnett AL, CanbyHagino ED, Cookson MS, et al.: Guideline for the management of clinically localized prostate cancer: 2007 update. J Urol. 2007; 177: 2106-31.

10. Heidenreich A, Aus G, Bolla M, Joniau S, Matveev VB, Schmid HP, et al.: EAU guidelines on prostate cancer. Eur Urol. 2008; 53: 68-80.

11. Hirobe M, Takahashi A, Hisasue S, Kitamura H, Kunishima Y, Masumori N, et al.: Bone scanning--who needs it among patients with newly diagnosed prostate cancer? Jpn J Clin Oncol. 2007; 37: 788-92.

12. Abuzallouf S, Dayes I, Lukka H: Baseline staging of newly diagnosed prostate cancer: a summary of the literature. J Urol. 2004; 171: 2122-7.

13. National Institute for Clinical Excellence. Guidance on Cancer Services: Improving Outcomes in Urological Cancers. The Manual National institute for Health and Clinical Excellence Website. available at http://www. nice.org.uk/nicemedia/pdf/Urological_Manual.pdf. Updated September.

14. Wymenga LF, Boomsma JH, Groenier K, Piers DA, Mensink HJ: Routine bone scans in patients with 
prostate cancer related to serum prostate-specific antigen and alkaline phosphatase. BJU Int. 2001; 88: 226-30.

15. Oesterling JE, Martin SK, Bergstralh EJ, Lowe FC: The use of prostate-specific antigen in staging patients with newly diagnosed prostate cancer. JAMA. 1993; 269: 57-60

16. Wolff JM, Zimny M, Borchers H, Wildberger J, Buell $\mathrm{U}$, Jakse G: Is prostate-specific antigen a reliable marker of bone metastasis in patients with newly diagnosed cancer of the prostate? Eur Urol. 1998; 33: 376-81.

17. Miller PD, Eardley I, Kirby RS: Prostate specific antigen and bone scan correlation in the staging and monitoring of patients with prostatic cancer. Br J Urol. 1992; 70: 295-8.

18. Kosuda S, Yoshimura I, Aizawa T, Koizumi K, Akakura K, Kuyama J, et al.: Can initial prostate specific antigen determinations eliminate the need for bone scans in patients with newly diagnosed prostate carcinoma? A multicenter retrospective study in Japan. Cancer. 2002; 94: 964-72.

19. Lee N, Fawaaz R, Olsson CA, Benson MC, Petrylak DP, Schiff PB, et al.: Which patients with newly diag- nosed prostate cancer need a radionuclide bone scan? An analysis based on 631 patients. Int J Radiat Oncol Biol Phys. 2000; 48: 1443-6.

20. Gleave ME, Coupland D, Drachenberg D, Cohen L, Kwong S, Goldenberg SL, et al.: Ability of serum prostate-specific antigen levels to predict normal bone scans in patients with newly diagnosed prostate cancer. Urology. 1996; 47: 708-12.

21. Coffey DS: Similarities of prostate and breast cancer: Evolution, diet, and estrogens. Urology. 2001; 57(4 Suppl 1): 31-8.

22. Zhu YP, Ye DW, Yao XD, Zhang SL, Dai B, Zhang HL, et al.: Prevalence of incidental prostate cancer in patients undergoing radical cystoprostatectomy: data from China and other Asian countries. Asian J Androl. 2009; 11: 104-8.

23. Chybowski FM, Keller JJ, Bergstralh EJ, Oesterling JE: Predicting radionuclide bone scan findings in patients with newly diagnosed, untreated prostate cancer: prostate specific antigen is superior to all other clinical parameters. J Urol. 1991; 145: 313-8.
Accepted after revision:

June 7, 2010

\section{Correspondence address:}

Dr. Mohammed A. Al-Ghazo

Department of General Surgery and Urology

Faculty of Medicine

Jordan University of Science and Technology

PO Box 3030, Irbid, 22110, Jordan

Fax: + 9622 720-0621

E-mail: alghazo@just.edu.jo

\section{EDITORIAL COMMENT}

The authors report about the bone scanning of newly diagnosed prostate cancer patients. This is an interesting retrospective study because the current guidelines for performing bone scans are based on limited historical data. They concluded that bone scan could be omitted in patients with a PSA level under $20 \mathrm{ng} / \mathrm{mL}$ and Gleason score under 8.

Radionuclide bone scan is the most sensitive modality for the detection of bone metastases. However, bone metastasis at diagnosis is not common 
in men without bone pain in the PSA screening era, the routine use of bone scans may not be useful nor be cost-effective. Recently, Briganti et al. performed external validation of currently available guidelines and concluded that staging bone scan might be considered for the patients with a biopsy Gleason score $>7$ or with a PSA $>10 \mathrm{ng} / \mathrm{mL}$ and palpable disease (cT2/T3) prior to local therapy.

To apply this retrospective data clinically, it is necessary to keep in mind prior to treatment that prostate cancer patients are at risk for bone metastasis. In addition, bone scan provides a baseline evaluation for comparison in men who later may complain of bone pain.

\section{REFERENCE}

1. Briganti A, Passoni N, Ferrari M, Capitanio U, Suardi N, Gallina A, et al.: When to Perform Bone Scan in Patients with Newly Diagnosed Prostate Cancer: External Validation of the Currently Available Guidelines and Proposal of a Novel Risk Stratification Tool. Eur Urol. 2010; 57: 551-8.

Dr. Taek Won Kang Department of Urology Chonnam National University Medical School Gwangju, Republic of Korea E-mail:sydad@hanmail.net 